\title{
Editorial: Malassezia: A Skin Commensal Yeast Impacting Both Health and Disease
}

\author{
Salomé LeibundGut-Landmann ${ }^{1,2 *}$ and Thomas L. Dawson $\mathrm{Jr}^{3,4}$ \\ 1 Section of Immunology, Vetsuisse Faculty, University of Zürich, Zürich, Switzerland, ${ }^{2}$ Institute of Experimental Immunology, \\ University of Zürich, Zürich, Switzerland, ${ }^{3}$ Skin Research Institute of Singapore, Agency for Science, Technology and \\ Research, Singapore, Singapore, ${ }^{4}$ Department of Drug Discovery, School of Pharmacy, Medical University of South Carolina, \\ Charleston, SC, United States
}

Keywords: Malassezia, commensalism and symbiosis, pathogenicity determinants, skin pathologies, new tools

Editorial on the Research Topic

Malassezia: A Skin Commensal Yeast Impacting Both Health and Disease

Over the last 30 years the term "microbiome" has grown exponentially in scientific discourse. Although the human-associated microbial community consists of bacteria, fungi, viruses, and archaea, the focus has been mainly on bacteria. Interest in the fungal communities, the mycobiome, has risen only more recently. In the skin, fungi represent $5 \%-10 \%$ of metagenomic sequences, versus less than $1 \%$ in mucosal epithelia (gut), and is dominated by a single fungal genus, Malassezia. Originally thought a single species, Malassezia is now known to span an entire clade comprising 18 diverse species and numerous functionally distinct strains. The lipid-dependent basidiomycetous yeast is found on all humans and warm-blooded vertebrates, and in ecosystems as diverse as deep marine environments. The high proportion of Malassezia in the skin microbiome makes understanding this fungus' role at the intersection with the host, the environment, and the microbiota crucial. Importantly, beyond the commensal lifestyle on host skin, Malassezia is also associated with various pathological conditions, including dandruff, pityriasis versicolor, and common inflammatory skin disorders such as seborrheic dermatitis and atopic dermatitis. While in some cases disease is linked to the appearance of Malassezia hyphae and fungal overgrowth, the causal relationship between Malassezia and disease often remains unclear. Fungal pathogenicity is defined as a relationship where the fungus may directly harm the host by specific virulence factors, or indirectly via induction of a harmful host response. New pathogenic roles of Malassezia beyond the skin have been revealed as Malassezia is associated with Crohn's disease and pancreatic ductal carcinoma. The present Special Topic of Frontiers in Fungal Pathogenesis makes a significant contribution to understanding more of the above-mentioned topics. The issue contains 13 diverse scientific contributions including 7 review and 6 primary research articles.

Vijaya Chandra et al. provide an up to date, comprehensive overview of Malassezia in human skin from early life to adulthood, including the importance of intact epithelial barriers and local immune system activity for stable colonization. However, how the Malassezia-host interaction transitions from a commensal to a pathogenic relationship remains a conundrum. The authors elaborate on whether Malassezia is cause or consequence in the multifaceted interaction with skin in 
health and under various dermatological conditions. They also discuss evidence for a protective, mutualistic, role of Malassezia in healthy skin through interaction with skin bacteria, especially atopic dermatitis-associated $S$. aureus, by restricting the bacteria's pathogenicity. In addition to skin, Malassezia is found in other unrelated niches. The detection in healthy faecal samples by sequencing and culture indicates that viable Malassezia occupies the gastrointestinal niche. Spatz and Richard expand on the adaptive flexibility of Malassezia to grow under conditions that differ from skin with respect to lipid content, temperature, and oxygen. Fungal dysbiosis is a hallmark of intestinal inflammation and as such an increase in the abundance of Malassezia in the gut mycobiome is associated with IBD. Spatz and Richard also speculate on mechanisms for how Malassezia is involved in gut pathologies. Beyond the involvement in skin disease and to a lesser degree other barrier tissue, Malassezia causes severe systemic infections, especially among premature neonates and immunocompromised patients receiving parenteral nutrition. Although rare, their occurrence is underestimated and their outcome often fatal. Rhimi et al. review the latest about the epidemiology and pathogenesis of Malassezia-related fungemia.

Malassezia is not restricted to humans, but also found in diverse vertebrate species. Guillot and Bond discuss the relevance of Malassezia in veterinary dermatology, from original discovery of $M$. pachydermatis being an important canine otic pathogen to the recent discovery of $M$. vespertilionis in bats. They highlight how management of Malassezia otitis externa and Malassezia dermatitis, commonly presented to veterinarians in small animal practice, relies on both treating fungal overgrowth and restoring host immunity, as the latter is often a predisposing factor for fungal overgrowth. Successful management of Malasseziarelated disease in humans and companion animals is hampered by poor diagnostics. Culture based isolation and enumeration of Malassezia remains challenging due to the lipid dependency. Saunte et al. review currently available diagnostic tools, including newer molecular methods, and treatment options for various skin pathologies. Identification of the involved Malassezia species is relevant for choosing the most effective antifungal drug, as different Malassezia spp. have varied antifungal susceptibility. The authors emphasize the need for standardization of species diagnostics and susceptibility testing. The challenges regarding robust and reproducible detection methods, treatment options and emerging resistance is also discussed by Rhimi et al. in the context of Malassezia fungemia and by Guillot and Bond in the context of Malassezia-mediated veterinary dermatitis and otitis.

Advances in Malassezia research are dependent on development of new tools for culture, detection, and genetic manipulation of the yeast, as well as for studying its pathogenicity and interaction with the mammalian host. Genome information is becoming available for an increasing number of species and strains, as summarized by Ianiri and Heitman and by Vijaya Chandra et al. Analysis of the available genomes contributed to resolving Malassezia taxonomy, shedding light on the evolutionary trajectory of Malassezia pathogenesis and niche adaptation, and enabling the identification of unknown genes and pathways linked to commensalism and/or pathogenicity. The availability of Malassezia genomes led to investigations considering emergence of Malassezia drug resistance. Park et al. assessed the molecular mechanisms that confer ketoconazole resistance, normally a highly effective fungistatic drug, in $M$. restricta. Comparative genome analysis of resistant strains isolated from dandruff patients suggests that multiplication of the genomic loci encoding genes involved in ergosterol synthesis and overexpression of drug efflux pumps are one mechanism underlying ketoconazole resistance in $M$. restricta. Genetic engineering of Malassezia was accomplished recently via agrobacterium-mediated transformation, as reviewed by Ianiri and Heitman. This powerful approach opens the door for both random insertional and targeted mutagenesis. The authors summarize the so far achieved mutants by either of the two approaches and discuss technical challenges and solutions. In addition, Goh et al. report generation of mCherry-expressing $M$. furfur via random insertional mutagenesis. Beyond fluorescently labelling yeast cells, this approach promises to be useful for overexpression of genes and the generation of insertional mutant libraries, which will help to deepen our understanding of Malassezia gene functions and hostmicrobes interactions.

Complementary to the new genetic tools, proteomic, metabolomic, and lipidomic approaches are essential for characterization of Malassezia species and strains in different environments. In this regard, Celis Ramírez et al. performed a lipidomic analysis and identified 18 lipid classes and 428 lipidic compounds, some of which segregate between different Malassezia species and may thus guide species discrimination and improve understanding of Malassezia lipid metabolism.

To study host-fungal interactions and assess antifungal immune responses, various models have become available. Studies with isolated cells in culture, including transformed keratinocyte and macrophage cell lines or primary cells isolated ex vivo, were recently complemented with approaches employing model hosts such as Galleria mellonella or experimental mice to explore the fungus-host interactions in vivo. In this Topic, Sparber et al. discuss the relevance of specific immune pathways for fungal control in barrier tissues. Type 17 immunity plays a central role in maintaining fungal homeostasis in healthy skin. However, IL-17 can exert pathological effects and exacerbate skin inflammation in barrier-disrupted skin. What determines the decision between protective and disease-promoting host response remains elusive. Also in this Topic, Corzo-León et al. describe an ex vivo human skin model, which provides a new and powerful approach to assess the host response to Malassezia of intact and fully differentiated human skin. The availability of diverse models opens the door for studying fungal pathogenesis and examining the functional role of putative virulence factors. As such, Vallhov et al. explored the host response to extracellular vesicles released from $M$. sympodialis and report activation of primary human keratinocytes, which strengthens the idea that extracellular vesicles play an important role in inter-organismal communication and initiation of the host response. The relevance of Malassezia-released compounds in the fungus-host interaction is further highlighted by Poh et al., which 
on the basis of genomic information explored secreted proteases in $M$. furfur and identified secreted aspartyl protease 1 (MfSAP1) as the dominant member of a family of 14 secreted proteases. They show that MfSAP1 is capable of degrading a wide range of human skin associated extracellular matrix proteins with important implication for cutaneous wound healing.

The diversity of contributions in this Special Topic shows that we are only scratching the surface to understanding Malassezia biology and the complexities of Malassezia-host interactions. A causative relationship between the fungus and various pathologies remains a matter of debate and the mechanism of pathogenesis unclear. Increased understanding of host- and microbe-specific interactions should lead to identification of key factors that maintain healthy skin homeostasis or, in turn, initiate pathogenic changes. Recent developments have expanded our understanding of Malassezia's role in the skin microbiome, with a focus on its multiple roles as commensal, pathogen, and protector through interactions with the host and inter-kingdom interactions. These approaches are leading towards development of new therapeutic targets and treatment options.

The guest editors would like to thank all the authors who submitted their work to the Special Topic, and all the reviewers for their hard work in completing timely and constructive reviews. Special thanks also go to the Editor-in-Chiefs and members of the editorial team for their support during the editing process.

\section{AUTHOR CONTRIBUTIONS}

SL-L drafted the manuscript. All authors contributed to the article and approved the submitted version.

\section{FUNDING}

The authors would like to acknowledge funding from the $A^{\star}$ STAR Industry alignment fund (H18/01/a0/016, to TLD) and the Swiss National Science Foundation (310030_189255, to SL-L).

Conflict of Interest: The authors declare that the research was conducted in the absence of any commercial or financial relationships that could be construed as a potential conflict of interest.

Copyright (C) 2021 LeibundGut-Landmann and Dawson. This is an open-access article distributed under the terms of the Creative Commons Attribution License (CC BY). The use, distribution or reproduction in other forums is permitted, provided the original author(s) and the copyright owner(s) are credited and that the original publication in this journal is cited, in accordance with accepted academic practice. No use, distribution or reproduction is permitted which does not comply with these terms. 